\title{
On the Rate of Asymptotic Eigenvalue Degeneracy
}

\author{
Evans M. Harrell * \\ Institut für theoretische Physik der Universität Wien, A-1090 Wien, Austria
}

\begin{abstract}
The gap between asymptotically degenerate eigenvalues of onedimensional Schrödinger operators is estimated. The procedure is illustrated for two examples, one where the solutions of Schrödinger's equation are explicitly known and one where they are not. For the latter case a comparison theorem for ordinary differential equations is required. An incidental result is that a semiclassical (W-K-B) method gives a much better approximation to the logarithmic derivative of a wave-function than to the wave-function itself; explicit error-bounds for the logarithmic derivative are given.
\end{abstract}

\section{Introduction}

The operator

$$
\begin{aligned}
H(\beta) & =-\frac{d^{2}}{d x^{2}}+x^{2}-2 \beta x^{3}+\beta^{2} x^{4} \\
& =-\frac{d^{2}}{d x^{2}}+x^{2}(1-\beta x)^{2}
\end{aligned}
$$

on $L^{2}(\mathbb{R})$, with $\beta \geqq 0$, and other similar operators have been studied because they exhibit asymptotic eigenvalue degeneracy as $\beta \rightarrow 0[1,2,3]$. The operator $H(\beta)$ is essentially self-adjoint on $\mathscr{S}(\mathbb{R})$. As $\beta \rightarrow 0$, it approaches the harmonic oscillator Hamiltonian, $H(0)=-\frac{d^{2}}{d x^{2}}+x^{2}$ (in the strong operator sense), and it is known that two distinct eigenvalues of $H(\beta)$ converge to each eigenvalue of $H(0)$. For example, the two lowest eigenvalues of $(1.1), E_{0}(\beta)$ and $E_{1}(\beta)$, both converge to $E_{0}(0)=1$ [the Number 1 will frequently be called $E_{0}(0)$, so that it will be clear how the formulae generalize]; and in fact both $E_{0}(\beta)$ and $E_{1}(\beta)$ have the same asymptotic - but almost certainly not convergent-power-series expansions in $\beta$,

* Present address: Department of Mathematics, MIT, Cambridge, MA 02139, USA; (National Needs Fellow) 
given by the Rayleigh-Schrödinger formula [4,5]. For $\beta>0, E_{0}(\beta)$ and $E_{1}(\beta)$ are certainly different, and Dirichlet-Neumann bracketing techniques have been used to show that

$$
C_{L} \exp \left(-k_{L} \beta^{-2}\right) \leqq E_{1}(\beta)-E_{0}(\beta) \leqq C_{U} \exp \left(-\mathrm{k}_{U} \beta^{-2}\right)
$$

for suitable positive constants $C_{L}, C_{U}, k_{L}$, and $k_{U}$ [3]. Of course, the function $C \exp \left(-k \beta^{-2}\right)$ has an asymptotic Taylor series at $\beta=0$ that is identically zero.

The existence of long-range order, and hence of phase transitions, in statistical systems is generally equivalent to the degeneracy or asymptotic degeneracy of the principle eigenvalue of some linear operator; for a discussion of this see the article by Kac [6], and for field-theory aspects see [7]. A new proof of the asymptotic degeneracy of the eigenvalues of (1.1) is implicit in the argument to be presented here. However, the emphasis is on constructing exact, calculable formulae to replace (1.2); in other words, on the exact critical behavior.

Operator (1.1) is invariant under the exchange $x \leftrightarrow(1 / \beta)-x$, i.e., reflection about $x=1 / 2 \beta$. By a well-known argument, the eigenfunctions $\psi_{\beta}^{n}$ such that $H(\beta) \psi_{\beta}^{n}=E_{n}(\beta) \psi_{\beta}^{n}$, and $E_{0}(\beta)<E_{1}(\beta)<\ldots, \beta>0$, satisfy

$$
\psi_{\beta}^{n}((1 / \beta)-x)=(-1)^{n} \psi_{\beta}^{n}(x), \quad n=0,1,2, \ldots
$$

The potential $x^{2}-2 \beta x^{3}+\beta^{2} x^{4}$ has two nearly harmonic wells, which are separated by ever greater distances and an ever higher barrier as $\beta \rightarrow 0$. A rough physical explanation for the asymptotic degeneracy is that the two wells asymptotically decouple into independent oscillators. The effects of a second well on the energy (eigenvalue) should be inversely proportional to the time a quantummechanical particle would take to tunnel through the barrier, by the uncertainty principle. Thus

$$
\begin{aligned}
\delta(\beta) \equiv & E_{1}(\beta)-E_{0}(\beta) \propto c \text { tunneling probability } \\
& \approx \exp \left(-2 \int_{t_{1}}^{t_{2}} \sqrt{x^{2}-2 \beta x^{3}+\beta^{2} x^{4}-E} d x\right),
\end{aligned}
$$

where $t_{1}$ and $t_{2}$ are the "classical turning points", the values of $x$ where $x^{2}-2 \beta x^{3}$ $+\beta^{2} x^{4}-E=0,0<x<1 / \beta$, and where $E$ should be roughly $E_{0}(0)$. This is in fact qualitatively correct, for

$$
\begin{aligned}
\delta(\beta) & =\text { const } \cdot \exp \left(-1 \int_{t_{1}}^{t_{2}} \sqrt{x^{2}-2 \beta x^{3}+\beta^{2} x^{4}-E} d x\right) \\
& =O\left(\exp \left(-K \beta^{-2}\right)\right) \text { for some } K,
\end{aligned}
$$

to the leading asymptotic order. The overall constant is calculated as $\frac{2}{\sqrt{\pi}} e^{-1 / 2}$ in (7.3), below. Note that the exact formula is proportional to the barrier penetration probability for only half the barrier.

\section{A Soluble Example}

The calculation of the rate of convergence of the asymptotically degenerate eigenvalues of (1.1) involves some functional estimates that might obscure the 
underlying simple idea. So let us first examine another operator with an asymptotic degeneracy, for which the solutions of the differential equation

$$
\left[-\frac{d^{2}}{d x^{2}}+V(x ; \beta)-E\right] \psi(x ; \beta, E)=0
$$

are known. This is the case for $x$ in the interval $[0,1 / \beta], \beta<1 / 2$, and

$$
V(x ; \beta)= \begin{cases}\beta, & 0 \leqq x \leqq 1 \\ F / \beta^{2}, & 1<x<(1 / \beta)-1 \\ \beta, & (1 / \beta)-1 \leqq x \leqq(1 / \beta),\end{cases}
$$

where $F$ is a constant and we impose Dirichlet boundary conditions at the end points, $\psi(0 ; \beta, E)=0=\psi(1 / \beta ; \beta, E)$. This is a rather typical square-well and -barrier potential from elementary quantum mechanics. The two square wells asymptotically decouple as the barrier between them rises and they get farther apart. $V$ is chosen as $\beta$ in the two wells just so that the perturbation series for the eigenvalues are not $E_{n}(0)+0+0+\ldots$. The potential has the same symmetry as in (1.1). It is trivial to determine that the eigenvalues are the solutions of two transcendental equations, which result from matching solutions of (2.1) at the natural boundaries $x=1$ and $(1 / \beta)-1$. The solution of $(2.1)$ on the interval $[0,1]$ with $\psi(0 ; \beta, E)=0$ is $\psi_{\ell}(x ; \beta, E)=A \sin (\sqrt{E-\beta} x)$. The solution in $[1,(1 / \beta)-1]$ that is symmetric about $x=1 / 2 \beta$ is $\psi_{s}(x ; \beta, E)=B \cosh \left(\sqrt{\left(F / \beta^{2}\right)-E}((1 / 2 \beta)-x)\right)$. The condition to be met is

$$
\left(\frac{d \psi_{\ell}}{d x}\right) /\left.\psi_{\ell}\right|_{x=1}=\left(\frac{d \psi_{s}}{d x}\right) /\left.\psi_{s}\right|_{x=1},
$$

so the even eigenvalues are the solutions of

$$
\left(\frac{1}{\sqrt{E-\beta}}\right) \tan (\sqrt{E-\beta})=\left(\frac{1}{\sqrt{\left(F / \beta^{2}\right)-E}}\right) \tanh \left(\sqrt{\left(F / \beta^{2}\right)-E}\left(\frac{1}{2 \beta}-1\right)\right)
$$

(the equation has been inverted for the convenience of making Taylor expansions below). Similarly, the antisymmetric solution in $[1,(1 / \beta)-1]$ is

$$
\psi_{a}(x ; \beta, E)=C \sinh \left(\sqrt{\left(F / \beta^{2}\right)-E}\left(\frac{1}{2 \beta}-x\right)\right),
$$

and the odd eigenvalues are the solutions of

$$
\left(\frac{1}{\sqrt{E-\beta}}\right) \tan (\sqrt{E-\beta})=\left(\frac{1}{\sqrt{\left(F / \beta^{2}\right)-E}}\right) \operatorname{coth}\left(\sqrt{\left(F / \beta^{2}\right)-E}\left(\frac{1}{2 \beta}-1\right)\right) .
$$

The series for the eigenvalues in powers of $\beta$ are obtained by making Taylor expansions in (2.3) and (2.4). But the tanh and coth functions in these two equations have the same series expansion in powers of $\beta$ and $E-E_{0}(0)$, viz.,

$$
\begin{aligned}
& \tanh \left(\sqrt{\left(F / \beta^{2}\right)-E}((1 / 2 \beta)-1)\right) \sim 1+0+0+\ldots \\
& \operatorname{coth}\left(\sqrt{\left(F / \beta^{2}\right)-E}((1 / 2 \beta)-1)\right) \sim 1+0+0+\ldots
\end{aligned}
$$


The two functions differ to $O\left(\exp \left(-2 \sqrt{\left(F / \beta^{2}\right)-E}((1 / 2 \beta)-1)\right)\right)$, which is $o\left(\beta^{m}\right)$ for all positive $m<\infty$. Thus the power-series solutions of (2.3) and (2.4) are identical. Specifically [using $E_{0}(0)=\pi^{2}$ ]

$$
\begin{array}{r}
G_{\ell}(E, \beta) \equiv \frac{1}{\sqrt{E-\beta}} \tan (\sqrt{E-\beta}) \sim O+\frac{1}{2 \pi^{2}}\left(E-\pi^{2}-\beta\right) \\
-\frac{3}{8 \pi^{4}}\left(E-\pi^{2}-\beta\right)^{2}+\ldots
\end{array}
$$

which must equal

$$
\begin{aligned}
G_{\left\{\begin{array}{l}
a \\
s\}
\end{array}\right.}(E, \beta) \equiv & \frac{1}{\sqrt{\left(F / \beta^{2}\right)-E}}\left\{\begin{array}{l}
\tanh \\
\operatorname{coth}
\end{array}\right\}\left(\sqrt{\left(F / \beta^{2}\right)-E}\left(\frac{1}{2 \beta}-1\right)\right) \sim \frac{\beta}{\sqrt{F-E \beta^{2}}} \\
& \sim O+\frac{1}{\sqrt{F}} \beta+O+\frac{E \beta^{3}}{2 F^{3 / 2}}+\ldots
\end{aligned}
$$

By writing $E \sim \sum_{j=0} c_{j} \beta^{j}$ and collecting terms, one finds

$$
E \sim \pi^{2}-\left(\frac{2 \pi^{2}}{\sqrt{F}}-1\right) \beta+\ldots
$$

for $E=E_{0}(\beta)$ or $E_{1}(\beta)$. Note that for $F<4 \pi^{4}$ the tunneling effect is strong enough to lower the energy for small $\beta$, but for $F>4 \pi^{4}$ it is not. An asymptotic expression for $\delta(\beta) \equiv E_{1}(\beta)-E_{0}(\beta)$ is obtained from (2.5) and (2.6) by treating the difference between the tanh and coth functions as a small variation. Fix $\beta>0$, and suppose that the exact solution $E_{0}(\beta)$ of $G_{t}(E, \beta)=G_{s}(E, \beta)$ is known. Then using Taylor's theorem about the point $E=E_{0}(\beta)$, we can expand the equation $G_{\ell}(E, \beta)=G_{a}(E, \beta)$ as

$$
\begin{aligned}
G_{\ell} & \left(E_{0}(\beta)\right)+\frac{\partial}{\partial E} G_{\left.\ell\right|_{E}=E_{0}(\beta)} \cdot\left(E-E_{0}(\beta)\right)+\ldots \\
& =G_{a}(E, \beta) \\
& =G_{s}(E, \beta) \cdot \operatorname{coth}^{2}\left(\sqrt{\left(F / \beta^{2}\right)-E}\left(\frac{1}{2 \beta}-1\right)\right) \\
& =\left\{G_{s}\left(E_{0}(\beta)\right)+\frac{\partial}{\partial E} G_{\left.s\right|_{E=E_{0}(\beta)}}+\ldots\right\} \operatorname{coth}^{2}\left(\sqrt{\left(F / \beta^{2}\right)-E}\left(\frac{1}{2 \beta}-1\right)\right) .
\end{aligned}
$$

Keeping terms to first order in $E-E_{0}(\beta)$, the solution $E_{1}$ of this equation is

$$
E_{1}=E_{0}+\frac{\operatorname{coth}^{2}\left(\left(\sqrt{\left(F / \beta^{2}\right)-E_{1}}\left(\frac{1}{2 \beta}-1\right)\right)-1\right) G_{s}\left(E_{0}\right)}{\frac{d}{d E}\left(G_{\ell}-G_{s}\right)_{\mid E=E_{0}}}+O\left(\left(E_{1}-E_{0}\right)^{2}\right),
$$


so

$$
\begin{aligned}
\delta(\beta) & =\frac{4 \beta}{\sqrt{F} \frac{d}{d E} G_{\ell \mid E=\pi^{2}, \beta=0}} \exp \left(-\int_{1}^{\frac{1}{\beta}-1} \sqrt{V(x, \beta)-E_{1}} d x\right)(1+O(\beta)) \\
& =\frac{8 \pi^{2}}{\sqrt{F}} \beta \exp (-\sqrt{F}(1 / \beta-2) / \beta)(1+O(\beta)) .
\end{aligned}
$$

If higher-order terms in the Taylor series are kept, $\delta(\beta)$ can be estimated to arbitrary order as $\exp (-\sqrt{F}(1 / \beta-2) / \beta)$ times a series in powers of $\beta$. The exponent is half the barrier penetration exponent.

\section{Outline of the Analogous Calculation for Operator (1.1)}

Henceforth we consider Equation (2.1) with $V=x^{2}-2 \beta x^{3}+\beta^{2} x^{4}$ and for $x$ on the whole real line. In this case there is no natural boundary at which to match solutions, so the arbitrary choice $x=0$ is made. Define $\psi_{\ell}, \psi_{s}$, and $\psi_{a}$ to be the nontrivial solutions of (2.1) with the conditions

$$
\begin{aligned}
& \lim _{x \rightarrow-\infty} \psi_{\ell}(x ; \beta, E)=0 \quad \text { (overall scaling not yet specified) } \\
& \psi_{s}\left(\frac{1}{2 \beta} ; \beta, E\right)=1 ; \quad \frac{d}{d x} \psi_{s}\left(\frac{1}{2 \beta} ; \beta, E\right)=0 \\
& \psi_{a}\left(\frac{1}{2 \beta} ; \beta, E\right)=0 ; \quad \frac{d}{d x} \psi_{a}\left(\frac{1}{2 \beta} ; \beta, E\right)=-1
\end{aligned}
$$

Then the eigenvalues of (1.1) for $\beta>0$ are given as the solutions of

$$
\frac{1}{\psi_{\ell}} \frac{d \psi_{\ell}(0 ; \beta, E)}{d x}=\frac{1}{\psi_{s}} \frac{d \psi_{s}(0 ; \beta, E)}{d x},
$$

and

$$
\frac{1}{\psi_{\ell}} \frac{d \psi_{\ell}(0 ; \beta, E)}{d x}=\frac{1}{\psi_{a}} \frac{d \psi_{a}(0 ; \beta, E)}{d x} .
$$

The functions $\frac{1}{\psi_{\ell}} \frac{d \psi_{\ell}}{d x}(0 ; \beta, E), \frac{1}{\psi_{s}} \frac{d \psi_{s}}{d x}(0 ; \beta, E)$, and $\frac{1}{\psi_{a}} \frac{d \psi_{a}}{d x}(0 ; \beta, E)$ are continuously differentiable in $E$ in a neighborhood of $E_{0}(0)$ and in $\beta$ in $[0, C)$ for some positive constant $C$. (In fact it is elementary that $\psi_{s}$, and $\psi_{a}$ are entire in $x$ and $E$, and that there exists a solution $\psi_{\ell}$ that is also entire in $x$ and $E$. It is possible to show analyticity in $\beta$ in a region of $\mathbb{C}$ containing $(0, C)$ and continuity as $\beta \rightarrow 0$ by following similar calculations for the harmonic oscillator $([8,9]$, see also $[10])$ after suitable scaling and translation of the variables.) Then (3.1) can be put in the form

$$
F(E, \beta) \equiv \frac{1}{\psi_{s}} \frac{d \psi_{s}}{d x}(0 ; \beta, E)-\frac{1}{\psi_{\ell}} \frac{d \psi_{\ell}}{d x}(0 ; \beta, E)=0,
$$


where $F$ is $C^{1}$ in both variables. Moreover, for small $\beta, \partial F / \partial E$ is nonzero. As $\beta \rightarrow 0$, Equation (2.1) becomes equivalent to Weber's equation, and its solutions are expressible in terms of parabolic cylinder functions $[11,12]$ :

$$
\psi_{\ell}(x ; 0, E)=D_{(E-1) / 2}(-\sqrt{2} x),
$$

and

$$
\psi_{s}(x ; 0, E)=\psi_{a}(x ; 0, E)=D_{(E-1) / 2}(\sqrt{2} x) .
$$

So

$$
\begin{aligned}
\partial F / \partial E_{\mid \beta=0} & =\frac{\partial}{\partial E}\left[\frac{\left(\partial D_{\frac{1}{2}(E-1)}(\sqrt{2} x) / \partial x\right)}{D_{\frac{1}{2}(E-1)}(\sqrt{2} x)}-\frac{\left(\partial D_{\frac{1}{2}(E-1)}(-\sqrt{2} x) / \partial x\right)}{D_{\frac{1}{2}(E-1)}(-\sqrt{2} x)}\right]_{x=0} \\
& =2 \sqrt{2} \frac{\partial}{\partial E}\left[\frac{\left(\partial D_{\frac{1}{2}(E-1)}(z) / \partial z\right.}{D_{\frac{1}{2}(E-1)}(z)}\right]_{z=0} .
\end{aligned}
$$

When $E=E_{0}(0)=1$, this is equal to $\sqrt{\pi} \neq 0$. By continuity in $E$ and $\beta, \partial F / \partial E$ is nonzero for nearby values of $E$ and $\beta$. Thus, by the implicit function Theorem (3.1) has a solution $E_{0}(\beta)$, where $d E_{0}(\beta) / d \beta=-(\partial F / \partial \beta) /(\partial F / \partial E)$.

To gauge the difference between the functions $\frac{1}{\psi_{s}} \frac{d \psi_{s}}{d x}$ and $\frac{1}{\psi_{a}} \frac{d \psi_{a}}{d x}$ requires what is essentially a Wronskian argument.

Claim. For any $x_{0}>x$ such that $\frac{1}{\psi_{s}} \frac{d \psi_{s}}{d x}$ and $\frac{1}{\psi_{a}} \frac{d \psi_{a}}{d x}$ are finite on $\left[x, x_{0}\right]$,

$$
\begin{aligned}
\Delta(x ; \beta, E) & \equiv \frac{1}{\psi_{s}} \frac{d \psi_{s}}{d x}-\frac{1}{\psi_{a}} \frac{d \psi_{a}}{d x} \\
& =\left(\frac{1}{\psi_{s}} \frac{d \psi_{s}}{d x}\left(x_{0}\right)-\frac{1}{\psi_{a}} \frac{d \psi_{a}}{d x}\left(x_{0}\right)\right) \exp \left(\int_{x}^{x_{0}}\left(\frac{\psi_{s}^{\prime}\left(x^{\prime} ; \beta, E\right)}{\psi_{s}\left(x^{\prime} ; \beta, E\right)}+\frac{\psi_{a}^{\prime}\left(x^{\prime} ; \beta, E\right)}{\psi_{a}\left(x^{\prime} ; \beta, E\right)}\right) d x^{\prime}\right) .
\end{aligned}
$$

Remark. Sections 5 and 6 will show how to evaluate this function. The important thing to know about it at this point is that it is extremely small when $x=0$.

Proof. By substitution from (2.1),

$$
\begin{gathered}
\frac{d}{d x} \Delta(x ; \beta, E)=\frac{1}{\psi_{s}} \frac{d^{2} \psi_{s}}{d x^{2}}-\left(\frac{1}{\psi_{s}} \frac{d \psi_{s}}{d x}\right)^{2}-\frac{1}{\psi_{a}} \frac{d^{2} \psi_{a}}{d x^{2}}+\left(\frac{1}{\psi_{a}} \frac{d \psi_{a}}{d x}\right)^{2} \\
=\left(\frac{1}{\psi_{a}} \frac{d \psi_{a}}{d x}\right)^{2}-\left(\frac{1}{\psi_{s}} \frac{d \psi_{s}}{d x}\right)^{2}=-\Delta(x ; \beta, E)\left(\frac{1}{\psi_{s}} \frac{d \psi_{s}}{d x}+\frac{1}{\psi_{a}} \frac{d \psi_{a}}{d x}\right),
\end{gathered}
$$

which is a trivial differential equation. The solution for $x<x_{0}$ is

$$
\Delta(x ; \beta, E)=\left(\frac{1}{\psi_{s}} \frac{d \psi_{s}}{d x}\left(x_{0}\right)-\frac{1}{\psi_{a}} \frac{d \psi_{a}}{d x}\left(x_{0}\right)\right) \exp \left(\int_{x}^{x_{0}}\left(\frac{\psi_{s}^{\prime}\left(x^{\prime} ; \beta, E\right)}{\psi_{s}\left(x^{\prime} ; \beta, E\right)}+\frac{\psi_{a}^{\prime}\left(x^{\prime} ; \beta, E\right)}{\psi_{a}\left(x^{\prime} ; \beta, E\right)}\right) d x^{\prime}\right)
$$

so long as $\frac{1}{\psi_{s}} \frac{d \psi_{s}}{d x}(y)-\frac{1}{\psi_{a}} \frac{d \psi_{s}}{d x}(y)$ exists for $x \leqq y \leqq x_{0}$. 
It will be shown below (7.2), by choosing $x_{0}$ correctly, that

$$
\Delta(0 ; \beta, E)=o\left(\beta^{m}\right) \text { for all } m<\infty,
$$

for $E$ near $E_{0}(0)$. Condition (3.2) for the eigenvalue $E_{1}(\beta)$ becomes

$$
F(E, \beta)-\Delta(0 ; \beta, E)=0 .
$$

Taylor's theorem implies that there exists $\beta^{\prime}=\beta+\Delta(0 ; \beta, E) /(\partial F / \partial \beta)+o(\Delta(0 ; \beta, E))$ such that $F(E, \beta)+\Delta(0 ; \beta, E)=F\left(E, \beta^{\prime}\right)$. Thus

$$
\begin{aligned}
E_{1}(\beta)=E_{0}\left(\beta^{\prime}\right) & =E_{0}(\beta)+\frac{d E_{0}}{d \beta}(\beta) \cdot\left(\beta^{\prime}-\beta\right)+o\left(\beta^{\prime}-\beta\right) \\
& =E_{0}(\beta)+\frac{(\partial F / \partial \beta)}{(\partial F / \partial E)} \cdot \frac{\Delta\left(0 ; \beta, E_{0}(\beta)\right)}{(\partial F / \partial \beta)}+o\left(\beta^{\prime}-\beta\right) \\
& =E_{0}(\beta)+\frac{\Delta\left(0 ; \beta, E_{0}(\beta)\right)}{(\partial F / \partial E)}+o\left(\Delta\left(0 ; \beta, E_{0}(\beta)\right)\right) .
\end{aligned}
$$

So

$$
\delta(\beta) \equiv E_{1}(\beta)-E_{0}(\beta)=\frac{\Delta\left(0 ; \beta, E_{0}(\beta)\right)}{\sqrt{\pi}}+o\left(\Delta\left(0 ; \beta, E_{0}(\beta)\right)\right)
$$

because as $\beta \rightarrow 0, E_{0}(\beta) \rightarrow E_{0}(0)=1$ and $\partial F / \partial E \rightarrow \sqrt{\pi}$.

\section{A Comparison Theorem}

In order to make the calculations just outlined one obviously needs to have a sufficiently good approximation for the solutions of (2.1) with the potential of (1.1), or more precisely a good approximation for the logarithmic derivatives of the solutions, $\frac{1}{\psi} \frac{d}{d x} \psi$. A complication is that it will be necessary to make different approximations for different values of $x$.

There are various approximations for the solutions of Schrödinger's equation, such as the $\mathrm{W}-\mathrm{K}-\mathrm{B}$ method, that are successfully used in numerical studies without always being justified mathematically. It turns out that a $\mathrm{W}-\mathrm{K}-\mathrm{B}$ type approximation is indeed appropriate for this problem, because it can be proved to give a good estimate of the logarithmic derivative of (2.1) in the region of interest. The necessary comparison theorem follows.

Theorem. Let $A(x)$ and $B(x)$ be continuously differentiable functions on a real interval $I=[y, z]$ (or, if $z<y, I=[z, y]$ ), and suppose that on $I$,

$$
u^{\prime \prime}(x)=A(x) u(x)
$$

where $u(x)$ never equals 0 and $0 \leqq u^{\prime}(x) / u(x)$ (or respectively $0 \geqq u^{\prime}(x) / u(x)$ ) for all $x$; and

$$
v^{\prime \prime}(x)=(A(x)+B(x)) v(x)
$$


and that $v^{\prime}(y) / v(y)=u^{\prime}(y) / u(y)$. Define $\Omega_{ \pm}(x)=-\left(\frac{u^{\prime}(x)}{u(x)}\right) \pm \sqrt{\left(\frac{u^{\prime}(x)}{u(x)}\right)^{2}+B(x)}$, and suppose that

a) $-\left(u^{\prime}(x) / u(x)\right)^{2}<B(x)$ for all $x \in I$; and

b) if $\Omega_{+}(x)$ has a (local or global) minimum $M$ at some point $x_{0}$, then $\Omega_{-}\left(x_{0}\right)$ $<\Omega_{+}\left(x_{0}\right)$, and $x>x_{0} \Rightarrow \Omega_{-}(x)<M$ (respectively $\left.b^{\prime}\right)$ if $\Omega_{-}(x)$ has a maximum $M$ at $x_{0}$, then $\Omega_{-}\left(x_{0}\right)<\Omega_{+}\left(x_{0}\right)$, and $\left.x<x_{0} \Rightarrow \Omega_{+}(x)>M\right)$.

Then

$$
\begin{aligned}
& u^{\prime}(z) / u(z)+\min \left\{0, \inf _{\xi \in I} \Omega_{+}(\xi)\right\} \\
& \quad \leqq v^{\prime}(z) / v(z) \\
& \quad \leqq u^{\prime}(z) / u(z)+\max \left\{0, \sup _{\xi \in I} \Omega_{+}(\xi)\right\} .
\end{aligned}
$$

(Respectively (4.3) holds with $\Omega_{-}$replacing $\Omega_{+}$.)

Remarks. 1. Similar bounds hold throughout $I$, by $z \rightarrow z^{\prime} \in I$.

2. In practice, the complicated-sounding condition b) is easy to verify; the proof shows why it is natural. It is implied by

c) $B(x) \geqq 0$ for all $x$, and $u^{\prime}(x) / u(x)=0 \Rightarrow B(x)>0$.

Then the conclusion of the theorem becomes simply

$$
u^{\prime} / u \leqq v^{\prime} / v \leqq u^{\prime} / u+\sup _{\xi \in I} \Omega_{+}(\xi)
$$

[respectively $\left.u^{\prime} / u \geqq v^{\prime} / v \geqq u^{\prime} / u+\inf _{\xi \in I} \Omega_{-}(\xi)\right]$.

On the other hand, if $B(x) \leqq 0$ for all $x$, then the conclusion also simplifies somewhat, because $\Omega_{ \pm}<0$ (respectively $\Omega_{ \pm}>0$ ).

In all cases of interest here, $B \ll\left(u^{\prime} / u\right)^{2}$, which means that

$$
\begin{aligned}
\Omega_{ \pm} & =-\left(\frac{u^{\prime}}{u}\right)\left(1 \mp \sqrt{1+B /\left[\frac{u^{\prime}}{u}\right]^{2}}\right) \\
& \cong\left\{\begin{array}{l}
B / 2\left[\frac{u^{\prime}}{u}\right] \\
-2 \frac{u^{\prime}}{u}
\end{array}\right] \text { respectively, } \Omega_{ \pm}=-\left(\frac{u^{\prime}}{u}\right)\left(1 \pm \sqrt{1+B /\left[\frac{u^{\prime}}{u}\right]^{2}}\right) \cong\left\{\begin{array}{l}
-2 u^{\prime} / u \\
B / 2\left[\frac{u^{\prime}}{u}\right]
\end{array}\right],
\end{aligned}
$$

simplifying condition b) considerably. In fact, usually

$\sup \Omega_{-}<\inf \Omega_{+} \cdot$

3. In this theorem, $u$ is to be thought of as a known function to which $v$ is compared, granting that they have the same boundary conditions at the point $y$, up to a scaling factor. 
4. The conditions of the theorem are sufficient but not minimal. All that is required is that the solutions of (4.1) and (4.2) are sufficiently differentiable for all the calculations in the proof to be defined, and that a) and b) hold. For a discussion of the smoothness of solutions of differential equations see, e.g., [13].

5. Moreover, the condition that $u^{\prime} / u=v^{\prime} / v$ at $y$ can be relaxed. In fact, if

$$
\sup _{\xi \in I} \Omega_{-}(\xi)<v^{\prime}(y) / v(y)-u^{\prime}(y) / u(y) \leqq \sup _{\xi \in I} \Omega_{+}(\xi)
$$

then

$$
\begin{aligned}
& \min \left\{v^{\prime}(y) / v(y)-u^{\prime}(y) / u(y), \inf _{\xi \in I} \Omega_{+}(\xi)\right\} \\
& \leqq v^{\prime}(z) / v(z)-u^{\prime}(z) / u(z) \\
& \leqq \sup _{\xi \in I} \Omega_{+}(\xi) .
\end{aligned}
$$

Respectively, if

$$
\inf _{\xi \in I} \Omega_{+}(\xi)>v^{\prime}(y) / v(y)-u^{\prime}(y) / u(y) \geqq \inf _{\xi \in I} \Omega_{+}(\xi),
$$

then

$$
\begin{aligned}
\max & \left\{v^{\prime}(y) / v(y)-u^{\prime}(y) / u(y), \sup _{\xi \in I} \Omega_{-}(\xi)\right\} \\
& \geqq v^{\prime}(z) / v(z)-u^{\prime}(z) / u(z) \\
& \left.\geqq \inf \Omega_{\xi \in I}(\xi) .\right]
\end{aligned}
$$

This follows from only a slight modification of the proof.

Proof. The case $z<y$, given by the respectively's in parentheses, follows simply by reflection of the coordinate from the more natural direction. Both directions are included because both are needed below. We may assume $y=0$. Define the function $w(x)$ so that $v(x)=w(x) u(x)$. Substitution into (4.2) and use of (4.1) readily yields the equation for $w$ :

$$
w^{\prime \prime}+2 w^{\prime}\left(\frac{u^{\prime}}{u}\right)-B(x) w=0 .
$$

Letting $\Omega(x)=w^{\prime}(x) / w(x)$, we then find

$$
\begin{aligned}
\Omega^{\prime}(x) & =w^{\prime \prime}(x) / w(x)-\left(w^{\prime}(x) / w(x)\right)^{2} \\
& =B(x)-2\left(u^{\prime}(x) / u(x)\right) \Omega-\Omega^{2} .
\end{aligned}
$$

The assumptions of the theorem imply that $\Omega(x)$ is continuously differentiable. Since $v^{\prime} / v=u^{\prime} / u+w^{\prime} / w=u^{\prime} / u+\Omega, v^{\prime}(0) / v(0)=u^{\prime}(0) / u(0) \Rightarrow \Omega(0)=0$. Without solving (4.4) with this boundary condition, it is elementary to determine where in the $\Omega x$ $x$-plane $d \Omega / d x$ would be positive, negative, or zero. The boundaries between these 
regions are the lines where $\Omega^{\prime}=0$, i.e., from (4.4) and the quadratic formula, at

$$
\Omega_{ \pm}(x) \equiv-u^{\prime}(x) / u(x) \pm \sqrt{\left(\frac{u^{\prime}(x)}{u(x)}\right)^{2}+B(x)} .
$$

Thus

$$
\begin{gathered}
\Omega(x)>\Omega_{+}(x) \Rightarrow \Omega^{\prime}(x)<0, \\
\Omega(x)<\Omega_{-}(x) \Rightarrow \Omega^{\prime}(x)<0, \quad \text { and } \\
\Omega_{-}(x)<\Omega(x)<\Omega_{+}(x) \Rightarrow \Omega^{\prime}(x)>0 .
\end{gathered}
$$

[And if $\Omega_{ \pm}(x)$ are not real, then $\Omega^{\prime}(x)$ is always negative.]

Since $B>-\left(u^{\prime}(x) / u(x)\right)^{2}$ by assumption, $\Omega_{ \pm}(x)$ are real for all $x$ and $\Omega_{+}(x)$ $>\Omega_{-}(x)$. Suppose that $\Omega(z)>\max \left\{0, \sup _{\xi \in I} \Omega_{+}(\xi)\right\}$. Since $\Omega \in C^{1}(I)$ and $\Omega(0)=0$, there exists $x<z$ such that $\Omega(x)>\max \left\{0, \sup _{\xi \in I} \Omega_{+}(\xi)\right\} \geqq \Omega_{+}(x)$ and $d \Omega(x) / d x>0$ (by the intermediate-value theorem). But this contradicts the first of relationships (4.5), so we conclude that

$$
\Omega(z) \leqq \max \left\{0, \sup _{\xi \in I} \Omega_{+}(\xi)\right\} .
$$

The upper bound of (4.3) then follows from $v^{\prime} / v=u^{\prime} / u+\Omega$.

The lower bound is similar but requires an extra step. Assume first that $\min \left\{0, \Omega_{+}(x)\right\}$ is a monotonically decreasing function, and suppose that for some $z^{\prime}, \Omega\left(z^{\prime}\right)<\min \left\{0, \inf _{\xi \in\left[y, z^{\prime}\right]} \Omega_{+}(\xi)\right\}$, which equals $\min \left\{0, \Omega_{+}\left(z^{\prime}\right)\right\}$. Since $\Omega(x) \in C^{1}(I)$ and $\Omega(0)=0$, and the function $\min \left\{0, \Omega_{+}(x)\right\}$ is also continuous, there must exist $z^{\prime \prime}<z^{\prime}$ such that $\Omega\left(z^{\prime \prime}\right)<\min \left\{0, \Omega_{+}\left(z^{\prime \prime}\right)\right\}$, and $\Omega^{\prime}\left(z^{\prime \prime}\right)<0$ again by the intermediate value theorem. In fact, $z^{\prime \prime}$ can clearly be chosen so that $\min \left\{0, \Omega_{+}\left(z^{\prime \prime}\right)\right\}-\Omega\left(z^{\prime \prime}\right)<\varepsilon$ for any $\varepsilon>0$, by continuity. Thus $z^{\prime \prime}$ can be chosen so that $\Omega\left(z^{\prime \prime}\right)>\Omega_{-}\left(z^{\prime \prime}\right)$, because, from its definition and the condition that $u^{\prime} / u>0, \Omega_{-}(x)$ is strictly less than $\min \left\{0, \Omega_{+}(x)\right\}$. But then the statement $\Omega^{\prime}\left(z^{\prime \prime}\right)<0$ contradicts the last of relationships (4.5), so we conclude that $\Omega(x) \geqq \min \left\{0, \inf _{\xi \in[y, x]} \Omega_{+}(\xi)\right\}$ as long as $\min \left\{0, \Omega_{+}\right\}$is monotonically decreasing between $y$ and $x$.

If $\min \left\{0, \Omega_{+}(x)\right\}$ is not monotonically decreasing throughout $I$, then it, and therefore also $\Omega_{+}(x)$, must attain a minimum, which is negative, at some point $x_{0}$. Assume the minimum is global. The uniqueness theorem for first-order differential equations of the type of (4.4) (here we again need some minimal smoothness) implies that since, as already established, $\Omega\left(x_{0}\right) \geqq \Omega_{+}\left(x_{0}\right)$, it follows for all $x>x_{0}$ that $\Omega(x)>\tilde{\Omega}\left(x_{0}\right)$, where $\tilde{\Omega}(x)$ satisfies (4.4) with the boundary condition $\tilde{\Omega}\left(x_{0}\right)$ $=\Omega_{+}\left(x_{0}\right)$.

Because $\Omega_{+}(x)>\Omega_{-}(x)$ for all $x$, continuity and (4.5) imply that throughout some interval $\left(x_{0}, x_{0}+r\right], r>0, \tilde{\Omega}^{\prime}(x)>0$, and thus $\tilde{\Omega}\left(x_{0}+r\right)>\Omega_{+}\left(x_{0}\right)$. By assumption b), $\Omega_{-}(x) \leqq \Omega_{+}\left(x_{0}\right)$ for all $x>x_{0}$. Thus for all $x>x_{0}$, the function $\tilde{\Omega}(x)$ either increases monotonically, or else $\tilde{\Omega}(x) \geqq \Omega_{+}(x)>\Omega_{+}\left(x_{0}\right)$ [because of (4.5)]. 


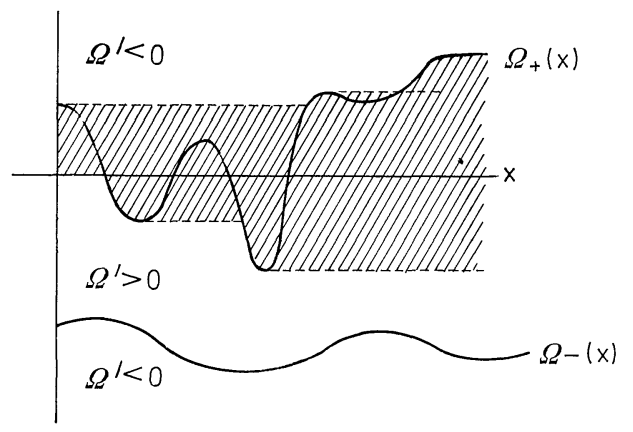

Fig. 1. Proof of the comparison theorem. By continuity of $\Omega$ and its derivative, $\Omega$ remains in the shaded region

Thus the bound

$$
\Omega(x) \geqq \min \left\{0, \inf _{\xi \in I} \Omega_{+}(\xi)\right\}
$$

holds, which implies the lower bound of (4.3) because $v^{\prime} / v=u^{\prime} / u+\Omega$. If $\Omega_{+}\left(x_{0}\right)$ is only a local minimum, then the above argument proves (4.3) in the subinterval of $I$ in which it is a global minimum, and it is necessary to repeat it for successive intervals.

The way this theorem will be used below is that comparison functions $u$ for $v=$ some solution of Schrödinger's equation will be found so that the appropriate $B(x)$ are small, which implies that the $\Omega_{+}$'s (or, as the case may be, the $\Omega_{-}$'s) are also small, and give useful error bounds.

\section{W-K-B is Fairly Accurate Where the Potential is Large}

The next two sections are devoted to deriving the estimate

$$
\begin{aligned}
& \frac{\psi_{s}^{\prime}(x ; \beta, E)}{\psi_{s}(x ; \beta, E)}=\left\{\begin{array}{l}
-x+O\left(\beta^{1-2 a}\right), \quad 0 \leqq x \leqq \beta^{-a} \\
-(V(x ; \beta)-E)^{1 / 2} \tanh \left(\int_{x}^{1 / 2 \beta}\left(V\left(x^{\prime} ; \beta\right)-E\right)^{1 / 2} d x^{\prime}\right) \\
-\frac{1}{4} \frac{d}{d x} \ln (V(x ; \beta)-E)+O\left(\beta^{2}\right)+O\left(x^{-3}\right), \quad \beta^{-a} \leqq x \leqq 1 / 2 \beta
\end{array}\right. \\
& \frac{\psi_{a}^{\prime}(x ; \beta, E)}{\psi_{a}(x ; \beta, E)}=\left\{\begin{array}{c}
-x+O\left(\beta^{1-2 a}\right), \quad 0 \leqq x \leqq \beta^{-a} \\
-(V(x ; \beta)-E)^{1 / 2} \operatorname{coth}\left(\int_{x}^{1 / 2 \beta}\left(V\left(x^{\prime} ; \beta\right)-E\right)^{1 / 2} d x^{\prime}\right) \\
-\frac{1}{4} \frac{d}{d x} \ln (V(x ; \beta)-E)+O\left(x^{-3}\right), \quad \beta^{-a} \leqq x \leqq 1 / 2 \beta
\end{array}\right.
\end{aligned}
$$

for all $a \in(0,1 / 4]$. The algebra is unfortunately rather involved, and straightforward but tedious steps will be omitted. When collecting error bounds it will often 
be understood that Taylor expansions have been made and higher-order terms dropped.

Let us first consider the intervals where the potential in (1.1) is large. Nonrigorous arguments lead one to expect that the $\mathrm{W}-\mathrm{K}-\mathrm{B}$ solutions in these regions should be reasonably accurate. Thus one expects $\psi$ to be approximately a linear combination of

$$
(V-E)^{-1 / 4} \exp \left(\int^{x}\left(V\left(x^{\prime} ; \beta\right)-E\right)^{1 / 2} d x^{\prime}\right)
$$

and

$$
(V-E)^{-1 / 4} \exp \left(-\int^{x}\left(V\left(x^{\prime} ; \beta\right)-E\right)^{1 / 2} d x^{\prime}\right)
$$

In particular, as $x \rightarrow-\infty$,

$$
\psi_{W, \ell}(x) \equiv(V-E)^{-1 / 4} \exp \left(-\int_{x}^{x_{0}}\left(V\left(x^{\prime} ; \beta\right)-E\right)^{1 / 2} d x^{\prime}\right)
$$

has the right asymptotic behavior. We will use this as the comparison function $(u)$ of the theorem for $v=\psi_{\ell}$. By substitution, $\psi_{W, \ell}^{\prime \prime}=A(x ; \beta, E) \psi_{W}$, where

$$
A(x ; \beta, E) \equiv V(x ; \beta)-E-B(x ; \beta, E),
$$

and

$$
B(x ; \beta, E) \equiv \frac{1}{4}(V-E)^{-1} V^{\prime \prime}-\frac{5}{16}(V-E)^{-2}\left(V^{\prime}\right)^{2} .
$$

Thus $\psi_{\ell}^{\prime \prime}=(A+B) \psi_{\ell}$, and

$$
\begin{aligned}
0<\psi_{W, \ell}^{\prime}(x ; \beta, E) / \psi_{W, \ell}(x ; \beta, E) & =(V-E)^{1 / 2}-\frac{1}{4} V^{\prime} /(V-E) \\
& =|x|\left((1-\beta x)^{2}-E / x^{2}\right)^{1 / 2}-\frac{x(1-\beta x)(1-2 \beta x)}{2\left(x^{2}(1-\beta x)^{2}-E\right)} .
\end{aligned}
$$

For calculational simplicity we make some crude estimates, assuming that $x \leqq-C<-\sqrt{E}$ :

$$
\begin{gathered}
\begin{aligned}
& \psi_{W, \ell}^{\prime}(x ; \beta, E) / \psi_{W, \ell}(x ; \beta, E) \geqq|x|\left(1-E / C^{2}\right)^{1 / 2}-\frac{1-2 \beta x}{2 x^{2}(1-\beta x)\left(1-E / C^{2}\right)} \\
& \geqq|x|\left(\left(1-E / C^{2}\right)^{1 / 2}-1 /\left(C^{3}-E C\right)\right) \\
&(\text { essentially }|x| \text { for large enough } C) ;
\end{aligned} \\
0>B(x ; \beta, E)=\frac{2\left(1-6 \beta x+6(\beta x)^{2}\right)}{4 x^{2}\left((1-\beta x)^{2}-E / x^{2}\right)}-\frac{5(1-\beta x)^{2}(1-2 \beta x)^{2}}{4 x^{2}\left((1-\beta x)^{2}-E / x^{2}\right)^{2}} \\
>-\frac{5 /\left(1-E / C^{2}\right)^{2}-2}{x^{2}} \\
\left(\text { essentially }-3 / x^{2}\right) .
\end{gathered}
$$

These expressions have been factorized for the convenience of the reader who wishes to check the straightforward algebra leading to the estimates. Recall that if 
$-1 \leqq z \leqq 0$, then $(1+z)^{1 / 2} \geqq 1+z$. Therefore, if $x$ is less than some constant (e.g., the lesser of -2 and $-10 \sqrt{E}$ ) then $0>B /\left(\psi_{W, \ell}^{\prime} / \psi_{W, \ell}\right)^{2} \geqq 1$, and for the appropriate $\Omega_{+}$,

$$
\begin{aligned}
0> & -\left(\psi_{W, \ell}^{\prime} / \psi_{W, \ell}\right)+\sqrt{\left(\psi_{W, \ell}^{\prime} / \psi_{W, \ell}\right)^{2}+B} \\
& =\left(\psi_{W, \ell}^{\prime} / \psi_{W, \ell}\right)\left(-1+\sqrt{1+B /\left(\psi_{W, \ell}^{\prime} / \psi_{W, \ell}\right)^{2}}\right. \\
& >B(x ; \beta, E) /\left(\psi_{W, \ell}^{\prime}(x ; \beta, E) / \psi_{W, \ell}(x ; \beta, E)\right) \geqq-\mathrm{const} /|x|^{3},
\end{aligned}
$$

where the constant is arbitrarily close to 3 for $C$ large enough. A similar calculation for $\Omega_{-}$shows that it is roughly $-2 \psi_{W, \ell}^{\prime} / \psi_{W, \ell}$, and so from (5.3) $C$ may be chosen so that $\sup _{-} \Omega_{-}(x)<\inf \Omega_{+}(x)$. Thus conditions a) and $\left.b\right)$ of the theorem are fulfilled on the interval $(-\infty, C]$. Letting $y \rightarrow-\infty$, we conclude

Proposition 1. If $\psi_{\ell}$ is the solution of (2.1) that behaves like $\psi_{W, \ell}$ as $x \rightarrow-\infty$, then for small enough $\beta$ and $x$ less than some negative constant, $-C$, and $C$ large enough,

$$
\begin{aligned}
& (V(x ; \beta)-E)^{1 / 2}-\frac{1}{4} V^{\prime}(x ; \beta) /(V(x ; \beta)-E) \\
& \quad \geqq \psi_{\ell}^{\prime}(x ; \beta, E) / \psi_{\ell}(x ; \beta, E) \\
& \quad \geqq(V(x ; \beta)-E)^{1 / 2}-\frac{1}{4} V^{\prime}(x ; \beta) /(V(x ; \beta)-E)-4 /|x|^{3} .
\end{aligned}
$$

Remark. Thus the $\mathrm{W}-\mathrm{K}-\mathrm{B}$ method estimates the logarithmic derivative of the wave function uniformly in $x$ to $O(1)$. But if $x$ is taken as a function of $\beta$, and $x \rightarrow-\infty$ as $\beta \rightarrow 0$, then the $\mathrm{W}-\mathrm{K}-\mathrm{B}$ method estimates the logarithmic derivative with a vanishingly small error, $O\left(x(\beta)^{-3}\right)$. The Number 4 is really $3+$ (function of $C)$. This estimate is not needed below, but is given for completeness and for illustrative purposes, as the calculation is simpler than in the other cases.

A similar argument allows the solutions of (2.1) that are symmetric and antisymmetric about the point $x=1 / 2 \beta$ to be estimated. The potential is large in the barrier and relatively flat near $x=1 / 2 \beta$, for

$$
x^{2}-2 \beta x^{3}+\beta^{2} x^{4}=1 / 16 \beta^{2}-\frac{1}{2}(x-1 / 2 \beta)^{2}+\beta^{2}(x-1 / 2 \beta)^{4} .
$$

The exponential $\mathrm{W}-\mathrm{K}-\mathrm{B}$ solutions do not have the proper symmetry, so we consider their symmetric and antisymmetric combinations.

$$
\psi_{W, s}(x ; \beta, E) \equiv(V-E)^{-1 / 4} \cosh \left(\int_{x}^{1 / 2 \beta}\left(V\left(x^{\prime} ; \beta\right)-E\right)^{1 / 2} d x^{\prime}\right),
$$

and

$$
\psi_{W, a}(x ; \beta, E) \equiv(V-E)^{-1 / 4} \sinh \left(\int_{x}^{1 / 2 \beta}\left(V\left(x^{\prime} ; \beta\right)-E\right)^{1 / 2} d x^{\prime}\right) .
$$

In the region of interest, $x<1 / 2 \beta$.

$$
\begin{aligned}
\psi_{W, s}^{\prime}(x, \beta, E) / \psi_{W, s}(x ; \beta, E)= & -(V(x ; \beta)-E)^{1 / 2} \tanh \left(\int_{x}^{1 / 2 \beta}\left(V\left(x^{\prime} ; \beta\right)-E\right)^{1 / 2} d x^{\prime}\right) \\
& -\frac{1}{4} V^{\prime}(x ; \beta) /(V(x ; \beta)-E),
\end{aligned}
$$


which is negative so long as $x<1 / 2 \beta$ and $V-E>0$, i.e., for $x \in[E+\varepsilon, 1 / 2 \beta), \varepsilon$ arbitrarily small.

Proposition 2. For small enough $\beta$ and $C \leqq x \leqq 1 / 2 \beta$, and $C$ large enough,

$$
\begin{gathered}
\psi_{W, s}^{\prime}(x ; \beta, E) / \psi_{W, s}(x ; \beta, E) \leqq \psi_{s}^{\prime}(x ; \beta, E) / \psi_{s}(x ; \beta, E) \\
\leqq \psi_{W, s}^{\prime}(x ; \beta, E) / \psi_{W, s}(x ; \beta, E)+\max \left\{5 \beta^{2}, 4 / x^{3}\right\} .
\end{gathered}
$$

Proof. It is actually necessary to make two separate estimates, because it turns out that in the vicinity of $x=1 / 2 \beta, \quad \psi_{s}$ is better approximated by $\varphi_{W, s} \equiv \cosh \left(\int_{x}^{1 / 2 \beta}\left(V\left(x^{\prime} ; \beta\right)-E\right)^{1 / 2} d x^{\prime}\right)$ than by $\psi_{W, s}=(V-E)^{-1 / 4} \varphi_{W, s}$. In the first step the subscript 1 will indicate that a given function is associated with $\varphi_{W, s}$, and in the second step the subscript 2 will indicate that $\psi_{W, s}$ is used.

Step 1. It is straightforward to calculate

$$
\begin{aligned}
\varphi_{W, s}^{\prime \prime} & =\left\{V-E-\frac{1}{2} \frac{V^{\prime}}{(V-E)} \tanh \left(\int_{x}^{1 / 2 \beta}\left(V\left(x^{\prime} ; \beta\right)-E\right)^{1 / 2} d x^{\prime}\right)\right\} \varphi_{W, s} \\
& \equiv A_{1}(x ; \beta, E) \varphi_{W, s} .
\end{aligned}
$$

We will use the comparison theorem with $v=\psi_{s}$ and $u_{1}=\varphi_{W, s}$; this means that

$$
B_{1}(x ; \beta, E)=\frac{1}{2} \frac{V^{\prime}}{(V-E)} \tanh \left(\int_{x}^{1 / 2 \beta}\left(V\left(x^{\prime} ; \beta\right)-E\right)^{1 / 2} d x^{\prime}\right) .
$$

Note that $B_{1} \geqq 0$ on the interval $[E+\varepsilon, 1 / 2 \beta), \varepsilon>0$ (and $=0$ only at $x=1 / 2 \beta$ ), and that

$$
0 \geqq \varphi_{W, s}^{\prime} / \varphi_{W, s}^{\prime}=-(V(x ; \beta)-E)^{1 / 2} \tanh \left(\int_{x}^{1 / 2 \beta}\left(V\left(x^{\prime} ; \beta\right)-E\right)^{1 / 2} d x^{\prime}\right) .
$$

Thus the estimate

$$
\varphi_{W, s}^{\prime}(x ; \beta, E) / \varphi_{W, s}(x ; \beta, E) \geqq \psi_{s}^{\prime}(x ; \beta, E) / \psi_{s}(x ; \beta, E)
$$

would follow from the theorem [unnatural direction, Remark 2, condition c)], with $y=1 / 2 \beta$, except that

$$
\varphi_{W, s}^{\prime} / \varphi_{W, s_{\mid x=1 / 2 \beta}}=0=B(1 / 2 \beta ; \beta, E),
$$

so one part of condition c) is violated. A limiting argument saves the estimate: Equation (5.7) implies that $\Omega_{1 \pm}(1 / 2 \beta)=0$. Since $\Omega_{1}(1 / 2 \beta)=0$, it is a priori possible that either

i) $\Omega_{1}(x) \geqq \Omega_{1}(x)$ as $x \rtimes 1 / 2 \beta$, or

ii) $0 \geqq \Omega_{1}(x) \geqq \Omega_{1-}(x)$ as $x \rtimes 1 / 2 \beta$,

because by the intermediate value theorem all other possibilities contradict (4.5) (see Fig. 2). If ii) holds, then for some $y^{\prime}<1 / 2 \beta$,

$$
\begin{aligned}
\inf _{x \leqq \xi \leqq y^{\prime}} \Omega_{1+}(\xi)>0>\Omega_{1}\left(y^{\prime}\right)= & \psi_{s}^{\prime}\left(y^{\prime} ; \beta, E\right) / \psi_{s}\left(y^{\prime} ; \beta, E\right) \\
& -\varphi_{W, s}^{\prime}\left(y^{\prime} ; \beta, E\right) / \varphi_{W, s}\left(y^{\prime} ; \beta, E\right)>\inf _{x \leqq \xi \leqq y^{\prime}} \Omega_{1-}(\xi),
\end{aligned}
$$




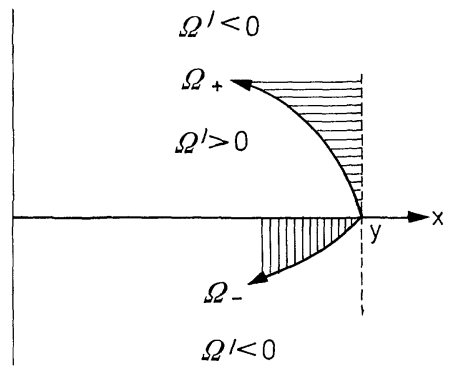

Fig. 2. Proof of Proposition 2. The function $\Omega(x)$ can not be in the upper shaded region because $\Omega(y)=0$, and $\Omega^{\prime}(y)=0$

and by Remark 5 of the theorem, the estimate (5.6) holds. But to see that i) is impossible, it is only necessary to calculate that $\Omega_{1+}^{\prime}(1 / 2 \beta)<0$, whereas from (4.4)

$$
\begin{aligned}
\Omega_{1}^{\prime}(1 / 2 \beta)= & B_{1}(1 / 2 \beta ; \beta, E) \\
& -2\left[\varphi_{W, s}^{\prime}(1 / 2 \beta ; \beta, E) / \varphi_{W, s}(1 / 2 \beta ; \beta, E)\right] \Omega_{1}(1 / 2 \beta)-\Omega_{1}^{2}(1 / 2 \beta)=0 .
\end{aligned}
$$

So as $x \gg 1 / 2 \beta, \Omega_{1}(x ; \beta, E)<\Omega_{1+}(x ; \beta, E)$.

Of course, the comparison theorem also gives a lower bound for $\psi_{s}^{\prime} / \psi_{s}$ complementary to (5.6), but it turns out not to be accurate enough for our purposes. Instead we make a second comparison, with $\psi_{W, s}$, the disadvantage of which is that $\Omega_{2 \pm}(x)$ are not real near $x=1 / 2 \beta$; as we shall see below, $\psi_{W, s}^{\prime}(1 / 2 \beta) / \psi_{W, s}(1 / 2 \beta)=0$, while the appropriate $B_{2}(1 / 2 \beta)<0$.

Step 2. This time we set $u_{2}=\psi_{W, s}$ for $v=\psi_{s}$, and calculate

$$
\psi_{W, s}^{\prime \prime}=\left(V-E+\frac{5}{16}\left(\frac{V^{\prime}}{V-E}\right)^{2}-\frac{1}{4} V^{\prime \prime} /(V-E)\right) \psi_{W, s} .
$$

Thus we may take

$$
A_{2}=V-E+\frac{5}{16}\left(\frac{V^{\prime}}{V-E}\right)^{2}-\frac{1}{4} V^{\prime \prime} /(V-E),
$$

and

$$
B_{2}=\frac{1}{4} V^{\prime \prime} /(V-E)-\frac{5}{16}\left(\frac{V^{\prime}}{V-E}\right)^{2} ;
$$

$A$ and $B$ are the same as for Proposition 1, but the interval is different. Useful crude bounds for small $\beta$ in this case are

$$
\begin{aligned}
0 & \geqq \psi_{W, s}^{\prime}(x ; \beta, E) / \psi_{W, s}(x ; \beta, E) \\
& =-(V-E)^{1 / 2} \tanh \left(\int_{x}^{1 / 2 \beta}\left(V\left(x^{\prime} ; \beta\right)-E\right)^{1 / 2} d x^{\prime}\right)-\frac{1}{4} V^{\prime} /(V-E),
\end{aligned}
$$

and

$$
0>B_{2}(x ; \beta, E)>-\left[\frac{2+5 /\left(1-E / C^{2}\right)}{4\left(1-E / C^{2}\right)}\right] \frac{1}{x^{2}},
$$


and this constant is essentially $-7 / 4$ for large $C$.

On the interval $[C, 1 / 2 \beta)$, if $\Omega_{2}(x) \leqq 0$, then

$$
\Omega_{2}^{\prime}(x)=B_{2}(x ; \beta, E)-2\left(\frac{\psi_{W, s}^{\prime}}{\psi_{W, s}}\right) \Omega_{2}(x)-\Omega_{2}^{2}(x)<0 .
$$

This is because $\Omega_{2-}(x)$ is easily seen from its definition and (5.8) and (5.9) to be positive whenever it is real on this interval. On the other hand, if $\Omega_{ \pm}(x)$ are not real, then $\Omega^{\prime}(x)$ is automatically negative regardless of the value of $\Omega(x)$. Thus, since $\Omega_{2}(1 / 2 \beta)=0$,

$$
C \leqq x<1 / 2 \beta \Rightarrow \Omega_{2}(x) \geqq 0 \Rightarrow \psi_{s}^{\prime} / \psi_{s} \geqq \psi_{W, s}^{\prime} / \psi_{W, s}
$$

(from the formula $v^{\prime} / v=u^{\prime} / u+\Omega$ ).

Combining this with (5.6) yields

$$
\psi_{W, s}^{\prime} / \psi_{W, s} \leqq \psi_{s}^{\prime} / \psi_{s} \leqq \varphi_{W, s}^{\prime} / \varphi_{W, s}=\psi_{W, s}^{\prime} / \psi_{W, s}+\frac{1}{4} V^{\prime} /(V-E) .
$$

Now set $y=1 / 2 \beta-1$ (arbitrarily). For small enough $\beta$, and $C \leqq x \leqq y$,

$$
B_{2}(1 / 2 \beta-1, \beta, E)>-\left[\psi_{W, s}^{\prime}(1 / 2 \beta-1, \beta, E) / \psi_{W, s}(1 / 2 \beta-1, \beta, E)\right]^{2},
$$

so $\Omega_{2 \pm}(x)$ are real, and the following estimates can be made:

$$
\begin{aligned}
& \text { a) } 0 \leqq \Omega_{2}(y) \leqq \frac{1}{4} V^{\prime}(y ; \beta, E) /(V(y ; \beta, E)-E)=4 \beta^{2}+O\left(\beta^{3}\right) \\
& \text { b) } \inf _{x \leqq \xi \leqq y} \Omega_{2+}(\xi)>\Omega_{2}(y) ; \\
& \text { c) } \begin{aligned}
\psi_{W, s}^{\prime} / \psi_{W, s}<- & (V-E)^{1 / 2}(1-\varepsilon) \leqq-\frac{1}{2} x \sqrt{1-E / c^{2}}, \varepsilon \text { arbitrarily small; } \\
\text { d) } \sup _{x \leqq \xi \leqq y} \Omega_{2-}(\xi) & =\sup _{x \leqq \xi \leqq y}\left(-\frac{\psi_{W, s}^{\prime}(\xi)}{\psi_{W, s}(\xi)}\right)\left(1-\sqrt{1+B_{2}(\xi) /\left[\frac{\psi_{W, s}^{\prime}(\xi)}{\psi_{W, s}(\xi)}\right]^{2}}\right) \\
& \leqq \sup _{x \leqq \xi \leqq y} B_{2}(\xi) /\left[\frac{\psi_{W, s}^{\prime}(\xi)}{\psi_{W, s}(\xi)}\right] \leqq \text { const } / x^{3}
\end{aligned}
\end{aligned}
$$

(again using $-1 \leqq z \leqq 0 \Rightarrow \sqrt{1+z} \geqq 1+z$ ). The constant is essentially $+7 / 2$ for large $C$.

Thus by Remark 5 to the comparison theorem and (5.10),

$$
0 \leqq \frac{\psi_{s}^{\prime}(x)}{\psi_{s}(x)}-\frac{\psi_{W, s}^{\prime}(x)}{\psi_{W, s}(x)} \leqq \max \left\{(4+\varepsilon) \beta^{2}, \text { const } / x^{3}\right\},
$$

from which the proposition follows $(4+\varepsilon<5,7 / 2<4)$.

The analogous argument for $\psi_{a}$ produces

Proposition 3. For small enough $\beta$ and $C \leqq x \leqq 1 / 2 \beta$, and $C$ large enough,

$$
\begin{aligned}
& \psi_{W, a}^{\prime}(x ; \beta, E) / \psi_{W, a}(x ; \beta, E) \leqq \psi_{a}^{\prime}(x ; \beta, E) / \psi_{a}(x ; \beta, E) \\
& \quad \leqq \psi_{W, a}^{\prime}(x ; \beta, E) / \psi_{W, a}(x ; \beta, E)+4 / x^{3} .
\end{aligned}
$$

Remark. We see from (3.3) that in fact the functions $\psi_{s}^{\prime} / \psi_{s}$ and $\psi_{a}^{\prime} / \psi_{a}$ are highly correlated for $x$ not near $1 / 2 \beta$, because the integral in (3.3) is a large negative quantity. It is not clear from Propositions 2 and 3 that they are so well correlated. 
Proof. This proposition is substantially the same as the previous one. Step 1, however, is not needed. We identify $u=\psi_{W, a}$ and $v=\psi_{a}$. A calculation shows that $A$ and $B$ are the same as the ones in Proposition 1 (or the $A_{2}$ and $B_{2}$ of Proposition 2). But now $\Omega_{ \pm}(x)$ are real throughout the interval, because

$$
0>\psi_{W, a}^{\prime} / \psi_{W, a}=-(V-E)^{1 / 2} \operatorname{coth}\left(\int_{x}^{1 / 2 \beta}\left(V\left(x^{\prime} ; \beta\right)-E\right)^{1 / 2} d x^{\prime}\right) \text {. }
$$

which $\rightarrow-\infty$ as $x \rightarrow 1 / 2 \beta$. This time one finds

$$
\lim _{x \rightarrow 1 / 2 \beta} \Omega_{+}(x)=\infty,
$$

and

$$
\begin{aligned}
0 \leqq \Omega_{-}(x) & =-\frac{\psi_{W, a}^{\prime}(x ; \beta, E)}{\psi_{W, a}(x ; \beta, E)}\left(1-\sqrt{1+B(x ; \beta, E) /\left[\frac{\psi_{W, a}^{\prime}(x ; \beta, E)}{\psi_{W, a}(x, \beta, E)}\right]^{2}}\right) \\
& \leqq B(x, \beta, E) /\left[\frac{\psi_{W, a}^{\prime}(x ; \beta, E)}{\psi_{W, a}(x ; \beta, E)}\right] \leqq \mathrm{const} / x^{3} .
\end{aligned}
$$

The proposition follows from the comparison theorem (see Remark 2) as before. (The constant is the same as before.)

\section{The Unperturbed Solution is Fairly Accurate Where the Perturbation is Small}

The two regions where the $\mathrm{W}-\mathrm{K}-\mathrm{B}$ approximations are accurate do not meet, so a different comparison must be made in the intervening region. As might be expected, the solutions of

$$
u^{\prime \prime}=\left(x^{2}-E\right) u
$$

are suitable for this purpose.

Proposition 4. Let $a \in(0,1 / 4)$, and consider $E$ such that $E-E_{0}(0)=0(\beta)$. For $0 \leqq x \leqq \beta^{-a}$,

$$
\psi_{s}^{\prime}(x ; \beta, E) / \psi_{s}(x ; \beta, E)=-x+O\left(\beta^{1-2 a}\right) .
$$

and

$$
\psi_{a}^{\prime}(x ; \beta, E) / \psi_{a}(x ; \beta, E)=-x+O\left(\beta^{1-2 a}\right) .
$$

For $-\beta^{-a} \leqq x \leqq 0$,

$$
\psi_{\ell}^{\prime}(x ; \beta, E) / \psi_{\ell}(x ; \beta, E)=-x+O\left(\beta^{1-2 a}\right) .
$$

The implied constants are independent of $x$ and $E$.

Proof. The comparison theorem and Proposition 2 will be used to prove (6.2). The proofs of (6.3) and (6.4) using the other propositions are similar. We know from the Rayleigh-Schrödinger theory (or from a preliminary but cruder calculation like the one given in this paper) that $E_{0}(\beta)-E_{0}(0)$ and $E_{1}(\beta)-E_{0}(0)$ are $O\left(\beta^{2}\right)$, so the restriction on $E$ is not serious. 
We work first on the interval $x \in\left[1, \beta^{-a}\right]$, avoiding $x=0$ for later convenience (so $\Omega_{ \pm}$are real). Let $u$ be as in (6.1) and $v=\psi_{s}$. The boundary condition is

$$
u^{\prime}\left(\beta^{-a}\right) / u\left(\beta^{-a}\right)=\frac{\psi_{s}^{\prime}\left(\beta^{-a}, \beta, E\right)}{\psi_{s}\left(\beta^{-a}, \beta, E\right)},
$$

the function $A$ is $x^{2}-E$, and thus $B$ is the perturbation, $-2 \beta x^{3}+\beta^{2} x^{4}<0$. $B(x, \beta, E) \rightarrow 0$ uniformly as $\beta \rightarrow 0$, when $x \leqq \beta^{-a}, a<1 / 3$.

Some facts about parabolic-cylinder functions are needed $[11,12]$. The solutions of $(6.1)$ are linear combinations of $u_{-}(x) \equiv D_{\frac{1}{2}(E-1)}(\sqrt{2} x)$ and $u_{+}(x) \equiv D_{-\frac{1}{2}(E+1)}(i \sqrt{2} x)$, which have the asymptotic expansions

$$
u_{-}(x) \sim \exp \left(-x^{2} / 2\right)(\sqrt{2} x)^{\frac{1}{2}(E-1)}\left\{1-\frac{(E-1)(E-3)}{16 x^{2}}+\ldots\right\},
$$

and

$$
u_{+}(x) \sim \exp \left(+x^{2} / 2\right)(i \sqrt{2} x)^{-\frac{1}{2}(E+1)}\left\{1+\frac{(E+1)(E+3)}{16 x^{2}}+\ldots\right\},
$$

the accuracy of which improves as $x \rightarrow \infty$ (uniformly in $E$ on a compact set). Normalizing so that $u(x)=u_{-}(x)+\eta u_{+}(x)$, and using Proposition 2 , we see that

$$
\begin{aligned}
u^{\prime}\left(\beta^{-a}\right) / u\left(\beta^{-a}\right)= & \frac{u_{-}^{\prime}\left(\beta^{-a}\right)+\eta u_{+}^{\prime}\left(\beta^{-a}\right)}{u_{-}\left(\beta^{-a}\right)+\eta u_{+}\left(\beta^{-a}\right)} \\
= & \left(V\left(\beta^{-a} ; \beta\right) \tanh \left(\int_{x}^{1 / 2 \beta}\left(V\left(x^{\prime}, \beta\right)-E\right)^{1 / 2} d x^{\prime}\right)\right. \\
& -\frac{1}{4} V^{\prime}\left(\beta^{-a} ; \beta\right) /\left(V\left(\beta^{-a} ; \beta\right)-E\right) \\
& +O\left(\beta^{2}\right)+O\left(\beta^{3 a}\right)=\beta^{-a}+O\left(\beta^{a}\right) .
\end{aligned}
$$

Another fact about the parabolic-cylinder functions (which could also be shown simply with the comparison theorem!) is

$$
u_{-}^{\prime}(x) / u_{-}(x)=-x+O((E-1) / x)
$$

and

$$
u_{+}^{\prime}(x) / u_{+}(x)=+x+O((E+1) / x)
$$

Substituting from (6.5) and (6.7) into (6.6) and solving for $\eta$ to leading order in $\beta$ yields

$$
\eta=O\left(\beta^{(2-E) a} \exp \left(-\beta^{-2 a}\right)\right), \text { i.e., } o\left(\beta^{n}\right) \text { for all } n<\infty .
$$

Using this and (6.5), for $x<\beta^{-a}$,

$$
\begin{aligned}
u^{\prime}(x) / u(x) & =\frac{u_{-}^{\prime}(x)+\eta u_{+}^{\prime}(x)}{u_{-}(x)+\eta u_{+}(x)} \\
& =\frac{u_{-}^{\prime}(x)}{u_{-}(x)}+O\left(\beta^{a}\left(\beta^{a} x\right)^{1-E} \exp \left(x^{2}-\beta^{-2 a}\right)\right)
\end{aligned}
$$

The rather complicated error term is $O\left(\beta^{a}\right)$ when $x=O\left(\beta^{-a}\right)$, but if $x<\beta^{-a^{\prime}}$, where $a^{\prime}<a$, then it is $o\left(\beta^{n}\right)$ for all $n<\infty$. For small $\beta$, (6.7) and (6.9) together imply that 
$u^{\prime} / u$ is nonzero and $|B(x ; \beta, E)| \ll\left(\frac{u^{\prime}(x)}{u(x)}\right)^{2}$ when $1 \leqq x \leqq \beta^{-a}$. Therefore

$$
\begin{aligned}
\sup _{1 \leqq x \leqq \beta^{-a}} \Omega_{-}(x) & =\sup _{1 \leqq x \leqq \beta^{-a}}\left(-\frac{u^{\prime}(x)}{u(x)}\left(1-\sqrt{1+B(x ; \beta, E) /\left[\frac{u^{\prime}(x)}{u(x)}\right]^{2}}\right)\right) \\
& \leqq \sup _{1 \leqq x \leqq \beta^{-a}}\left(B(x ; \beta, E) /\left[\frac{u^{\prime}(x)}{u(x)}\right]\right) \\
& =O\left(\sup _{1 \leqq x \leqq \beta^{-a}}\left(-2 \beta x^{2}+\beta^{2} x^{4}\right)\right)=O\left(\beta^{1-2 a}\right) .
\end{aligned}
$$

This is certainly less than

$$
\inf _{1 \leqq x \leqq \beta^{-a}} \Omega_{+}(x) \approx \inf \left(-2 \frac{u^{\prime}(x)}{u(x)}\right) \approx+2 x
$$

for small $\beta$. The conditions of the comparison theorem are satisfied, and hence

$$
u^{\prime}(x) / u(x) \leqq \psi_{s}^{\prime}(x ; \beta, E) / \psi_{s}(x ; \beta, E) \leqq u^{\prime}(x) / u(x)+O\left(\beta^{1-2 a}\right) .
$$

As remarked after (6.9), if we-restrict the interval now to $1 \leqq x \leqq \beta^{-a^{\prime}}, a^{\prime}<a$, $u^{\prime} / u=u_{-}^{\prime} / u_{-}+o\left(\beta^{n}\right)$ for all $n$, and by $(6.7)$, this means that

$$
\begin{aligned}
& \psi_{s}^{\prime}(x ; \beta, E) / \psi_{s}(x ; \beta, E) \\
& \quad=-x+O((E-1) / x)+O\left(\beta^{1-2 a^{\prime}}\right)=-x+O\left(\beta^{1-2 a^{\prime}}\right) .
\end{aligned}
$$

This establishes (6.2) for $x \geqq 1$. For $0 \leqq x \leqq 1$, recall that $\psi_{s}^{\prime} / \psi_{s}$ is analytic in $E$ and $C^{1}$ in $\beta$; therefore if $E-E_{0}(0)$ is $O(\beta)$,

$$
\begin{aligned}
& \psi_{s}^{\prime}(x ; \beta, E) / \psi_{s}(x ; \beta, E) \\
& \quad=\psi_{s}^{\prime}\left(x ; 0, E_{0}(0)\right) / \psi_{s}\left(x ; 0, E_{0}(0)\right)+O(\beta)=-x+O(\beta) .
\end{aligned}
$$

By taking the supremum over the compact set $[0,1]$, the $O(\beta)$ estimate holds independently of $x$.

Remark: the interval $0 \leqq x \leqq 1$ can also be handled with a more complicated comparison argument, without using analyticity.

\section{Conclusion}

Using the results of Sections 5 and 6, Equation (3.3) can be evaluated as a constant times an exponential function of $\beta$, with a higher-order error. Choose $x_{0}=(1 / 2 \beta)-\beta$. From Propositions $2-4$,

$$
\begin{aligned}
\frac{\psi_{s}^{\prime}\left(x_{0} ; \beta, E\right)}{\psi_{s}\left(x_{0} ; \beta, E\right)}-\frac{\psi_{a}^{\prime}\left(x_{0} ; \beta, E\right)}{\psi_{a}\left(x_{0} ; \beta, E\right)} & \\
= & \left(V\left(x_{0} ; \beta\right)-E\right)^{1 / 2}\left\{\operatorname{coth}\left(\int_{x_{0}}^{1 / 2 \beta}(V(x ; \beta)-E)^{1 / 2} d x\right)\right. \\
& \quad-\tanh \left(\int_{x_{0}}^{1 / 2 \beta}(V(x ; \beta)-E)^{1 / 2} d x\right)+O\left(\beta^{2}\right) \\
= & \frac{1}{4 \beta}\{\operatorname{coth}(1 / 4)-\tanh (1 / 4)\}+O\left(\beta^{2}\right)
\end{aligned}
$$


and

$$
\begin{aligned}
\int_{0}^{x_{0}}\left(\frac{\psi_{s}^{\prime}(x ; \beta, E)}{\psi_{s}(x ; \beta, E)}+\frac{\psi_{a}^{\prime}(x ; \beta, E)}{\psi_{a}(x ; \beta, E)}\right) d x \\
=-\beta^{-2 a}+O\left(\beta^{1-3 a}\right)-\int_{\beta^{-a}}^{x_{0}} d x(V(x ; \beta)-E)^{1 / 2} \\
\left.\quad \cdot \operatorname{coth}\left(\int_{x}^{1 / 2 \beta}\left(V\left(x^{\prime} ; \beta\right)-E\right)^{1 / 2} d x^{\prime}\right)+\tanh \left(\int_{x}^{1 / 2 \beta}\left(V\left(x^{\prime} ; \beta\right)-E\right)^{1 / 2} d x^{\prime}\right)\right\} \\
\quad-\frac{1}{2} \int_{\beta^{-a}}^{x_{0}} \frac{d}{d x}[\ln (V(x ; \beta)-E)] d x+O\left(\beta^{2 a}\right) \\
=-\beta^{-2 a}-2 \int_{\beta^{-a}}^{1 / 2 \beta-1}(V(x ; \beta)-E)^{1 / 2} d x-\frac{1}{2} \ln \left[\frac{V\left(x_{0} ; \beta\right)-E}{V\left(\beta^{-a} ; \beta\right)-E}\right] \\
\quad-\int_{1 / 2 \beta-1}^{x_{0}} d x(V(x ; \beta)-E)^{1 / 2}\left\{\operatorname{coth}\left(\int_{x}^{1 / 2 \beta}\left(V\left(x^{\prime} ; \beta\right)-E\right)^{1 / 2} d x^{\prime}\right)\right. \\
\left.\quad+\tanh \left(\int_{x}^{1 / 2 \beta}\left(V\left(x^{\prime} ; \beta\right)-E\right)^{1 / 2} d x^{\prime}\right)\right\}+O\left(\beta^{2 a}\right) \\
=-2 \int_{\beta^{-a}}^{1 / 2 \beta-1}(V(x ; \beta)-E)^{1 / 2} d x-\beta^{-2 a}-\frac{1}{2} \ln \left(\beta^{-2+2 a} / 16\right) \\
\quad-\int_{1 / 2 \beta-1}^{x_{0}} \frac{1}{4 \beta}\left\{\operatorname{coth}\left(\frac{1 / 2 \beta-x}{4 \beta}\right)+\tanh \left(\frac{1 / 2 \beta-x}{4 \beta}\right)\right\} d x+O\left(\beta^{2 a}\right)
\end{aligned}
$$

[after making a couple of Taylor expansions, and noting that the last integral is $O(1)]$.

\section{Thus}

$$
\begin{aligned}
\int_{0}^{x_{0}}\left(\frac{\psi_{s}^{\prime}(x ; \beta, E)}{\psi_{s}(x ; \beta, E)}+\frac{\psi_{a}^{\prime}(x ; \beta, E)}{\psi_{a}(x ; \beta, E)}\right) d x \\
=\left[-2 \int_{\beta^{-a}}^{1 / 2 \beta-1}(V(x ; \beta)-E)^{1 / 2} d x-\beta^{-2 a}-\ln \beta^{a}\right] \\
\quad+\ln (4 \beta)-\ln \left(\frac{\sinh (1 / 4 \beta)}{\sinh (1 / 4)}\right)-\ln \left(\frac{\cosh (1 / 4 \beta)}{\cosh (1 / 4)}\right)+O\left(\beta^{2 a}\right) .
\end{aligned}
$$

This can be somewhat simplified

$$
\begin{aligned}
\ln (\sinh (1 / 4 \beta)) & =\ln ((\exp (1 / 4 \beta)+\exp (-1 / 4 \beta)) / 2) \\
& =1 / 4 \beta-\ln 2+O(\exp (-1 / 2 \beta)) \\
& =\int_{1 / 2 \beta-1}^{1 / 2 \beta}(V(x ; \beta)-E)^{1 / 2} d x-\ln 2+O(\beta)
\end{aligned}
$$

and likewise

$$
\ln (\cosh (1 / 4 \beta))=\int_{1 / 2 \beta-1}^{1 / 2 \beta}(V(x ; \beta)-E)^{1 / 2} d x-\ln 2+O(\beta) .
$$


Moreover, the expression in brackets of (7.1) is independent of $a$ to $O\left(\beta^{2 a}\right)$, despite appearances. If $t_{1}(\beta)$ is the turning point near $x=\sqrt{E_{0}(0)}$, then $t_{1}^{2}=E_{0}(0)+O(\beta)$, and

$$
\begin{aligned}
\int_{t_{1}(\beta)}^{\beta^{-a}}(V(x ; \beta)-E)^{1 / 2} d x=\int_{t_{1}(\beta)}^{\beta^{-a}}\left[\left(x^{2}-E\right)^{1 / 2}+O\left(\beta^{1-a}\right)\right] d x \\
=\frac{\beta^{-a}}{2}\left(\beta^{-2 a}-E\right)^{1 / 2}-\frac{E}{2} \ln \left(\beta^{-a}+\sqrt{\beta^{-2 a}-E}\right) \\
\quad-\frac{t_{1}}{2}\left(t_{1}^{2}-E\right)^{1 / 2}+\frac{E}{2} \ln \left(t_{1}+\sqrt{t_{1}^{2}-E}\right) \\
=\frac{1}{2} \beta^{-2 a}-\frac{E_{0}(0)}{4}+O\left(\beta^{2 a}\right)+\frac{E_{0}(0)}{2} \ln \beta^{a}-\frac{E_{0}(0)}{2} \ln 2+O\left(\beta^{2 a}\right) \\
\quad+\frac{E_{0}(0)}{2} \ln \sqrt{E_{0}(0)}+O\left(\beta^{1 / 2}\right) .
\end{aligned}
$$

Substituting, and recalling $E_{0}(0)=1$,

$$
\begin{aligned}
& {\left[-2 \int_{\beta^{a}}^{1 / 2 \beta-1} d x(V(x ; \beta)-E)^{1 / 2}-\beta^{-2 a}-\ln \beta^{a}\right]} \\
& \quad=-2 \int_{t_{1}}^{1 / 2 \beta-1}(V(x ; \beta)-E)^{1 / 2} d x-\frac{1}{2}-\ln 2+O\left(\beta^{2 a}\right) .
\end{aligned}
$$

Finally,

$$
\begin{aligned}
\int_{0}^{x_{0}} & \left(\frac{\psi_{s}^{\prime}(x ; \beta, E)}{\psi_{s}(x ; \beta, E)}+\frac{\psi_{a}^{\prime}(x ; \beta, E)}{\psi_{a}(x ; \beta, E)}\right) d x \\
= & \left.-2 \int_{t_{1}}^{1 / 2 \beta}\left(V_{x} ; \beta\right)-E\right)^{1 / 2} d x+\ln (8 \beta)-\frac{1}{2} \\
& +\ln (\sinh (1 / 4) \cosh (1 / 4))+O\left(\beta^{2 a}\right) .
\end{aligned}
$$

Substituting into (3.3) yields

$$
\begin{aligned}
\Delta(0 ; \beta, E) & =2 e^{-1 / 2}\left\{\cosh ^{2}(1 / 4)-\sinh ^{2}(1 / 4)\right\} \exp \left(-2 \int_{t_{1}}^{1 / 2 \beta}(V(x ; \beta)-E)^{1 / 2} d x\right) \\
& =2 e^{-1 / 2} \exp \left(-2 \int_{t_{1}}^{1 / 2 \beta}(V(x ; \beta)-E)^{1 / 2} d x\right) .
\end{aligned}
$$

Therefore, by (3.5),

$$
\delta(\beta)=\frac{2}{\sqrt{\pi}} e^{-1 / 2} \exp \left(-2 \int_{t_{1}}^{1 / 2 \beta}(V(x ; \beta)-E)^{1 / 2} d x\right)\left\{1+O\left(\beta^{2 a}\right)\right\} .
$$

The exponent $a$ is still arbitrary but at most $1 / 4$, so we take $a=1 / 4$, making the error $O\left(\beta^{1 / 2}\right)$.

A similar formula can be determined for the rate of asymptotic eigenvalue degeneracy of most other non-pathological, one-dimensional Schrödinger oper- 
ators with asymptotic degeneracies coming from double-well potentials. The dominant part of the calculation will be similar to a barrier-penetration probability, and the overall constant will depend on the unperturbed eigenfunctions. It should be possible to generate higher orders in the perturbation expansion (barrier pen. $x$ power series in $\beta$ ) by

1) using $n$-th-order perturbed approximations of some kind for the eigenfunction where the perturbation is small, rather than the unperturbed eigenfunction, and

2) using more sophisticated semiclassical approximations to the solutions in the large-potential region.

Potentials that are only asymptotically symmetric should be susceptible of a similar treatment, with more involved algebra. It may also be possible to study the asymptotic degeneracy of higher eigenvalues, but the comparison theorem can not be applied in the neighborhoods of the zeroes of the unperturbed eigenfunctions.

On the other hand, it is not clear that the comparison theorem generalizes in a useful way to higher dimensions, except where a separation of variables can be effected. If the theorem could be extended, a formula like (7.2) would result for operators with asymptotic degeneracy, using a higher-dimensional semiclassical method.

Since the acceptance of this paper, the author has learned of an earlier calculation of the eigenvalue gap in the physics literature, using functional integral methods. The rigorous results of the method given above agree precisely with the results of $[14]$.

Acknowledgements. I am grateful to Barry Simon for suggesting this problem and for encouragement, and to Ricardo Schor for correcting a multiplicative factor and telling me about Ref. [14]. Parts of this research were completed while I enjoyed the hospitality of Haverford College and of the Navajo Nation, as well as of the University of Vienna.

\section{References}

1. Thompson,C.J., Kac,M.: Phase transitions and eigenvalue degeneracy of a one-dimensional anharmonic oscillator. Studies Appl. Math. 48, 257-264 (1969)

2. Isaacson, D. : Singular perturbations and asymptotic eigenvalue degeneracy. Commun. Pure Appl. Math. 29, 531-551 (1976)

3. Reed, M., Simon, B. : Methods of modern mathematical physics, Vol. 4. New York: Academic Press 1978

4. Kato,T.: Perturbation theory for linear operators. Die Grundlehren der mathematischen Wissenschaften, Vol. 132. Berlin-Heidelberg-New York: Springer-Verlag 1966

5. Reed,M., Simon,B.: Methods of modern mathematical physics, Vol. III. New York: Academic Press 1978

6. Kac,M.: Mathematical mechanisms of phase transitions. In: Brandeis University Summer Institute in Theoretical Physics 1966, Vol. 1 (eds. M. Chrétien, E. P. Gross, S. Deser). New York: Gordon and Breach 1968

7. Glimm,J., Jaffe,A., Spencer, T.: Phase transitions for $\varphi_{2}^{4}$ quantum fields. Commun. math. Phys. 45, $203-216(1975)$

8. Simon, B.: Coupling constant analyticity for the anharmonic oscillator. Ann. Phys. 58, 76-136 (1970)

9. Loeffel,J.J., Martin, A.: Propriétés analytiques des niveaux de l'oscillateur anharmonique et convergence des approximants de Padé. CERN Ref. TH. 1167 (1970) 
10. Hsieh,P.-F., Sibuya, Y.: On the asymptotic integration of second order linear ordinary differential equations with polynomial coefficients. J. Math. Anal. Appl. 16, 84-103 (1966)

11. Whittaker,E.T., Watson,G.N.: A course of modern analysis. Cambridge: Cambridge University Press 1969

12. Abramowitz, M., Stegun,I. A., (eds.): Handbook of mathematical functions. Washington : National Bureau of Standards 1964

13. Coddington,E.A., Levinson, N.: The theory of differential equations. New York: McGraw-Hill 1955

14. Gildener,E., Patrascioiu,A.: Pseudoparticle contributions to the energy spectrum of a onedimensional system. Phys. Rev. D 16, 423-430 (1977)

Communicated by A. Jaffe

Received November 7, 1977 